\title{
How do algae endosymbionts mediate for their coral host fitness under heat stress? A comprehensive mechanistic overview
}

\author{
Montaser Al-Hammad ${ }^{1}$, thamara Ferreira Silva ${ }^{1}$, Hussein Hussein ${ }^{1}$, Garima Saxena ${ }^{1}$, Luzia \\ Modolo $^{1}$, hildegard westphal ${ }^{1}$, and Mohamed Farag ${ }^{2}$ \\ ${ }^{1}$ Affiliation not available \\ ${ }^{2}$ Cairo University
}

May 16, 2021

\begin{abstract}
Climate change is considered as one of the biggest threats to coral reefs in the next 100 years. The most significant impact of climate change would be the rise in global seawater temperature. A critical turning point which determines the fate of coral reefs depends on how corals and their associated algae respond to the rise in seawater temperature. Symbiotic plasticity may help corals to survive such environmental threats. The zooxanthella Symbiodinium is classified into nine clades (A I), six of which are known to be coral symbionts. As reaction to thermal stress, symbionts are lost to the host by several mechanisms. Physiological models on the importance of nitrogen fixing bacteria, i.e., diazotrophs, for bleached corals indicate that diazotrophically derived nitrogen either allows corals to better withstand bleaching, or increases bleaching by generating unbalanced nutrient requirements that lead to phosphorous starvation of the Symbiodinium. Reactive oxygen species (ROS) triggered by symbiosis operation of the damaged photosynthetic machinery causes leaks in the host cell, where they overpower cellular antioxidant mechanisms and potentially damage the host tissue. Both symbiotic partners, however, have significant adaptations for managing ROS to mitigate against cell damage as illustrated herein. Such extensive compile of literature in this review suggest that physiological host plasticity and/or symbiotic components clearly plays a significant role in response to thermal stress that may also vary between different species of corals, as many corals may contain specific ecotypes or clades of zooxanthellae which may vary in their ability to withstand thermal stress.
\end{abstract}

\section{Hosted file}

How do algae endosymbionts Mol ecology format.pdf available at https://authorea.com/users/ 414087/articles/522218-how-do-algae-endosymbionts-mediate-for-their-coral-host-fitnessunder-heat-stress-a-comprehensive-mechanistic-overview 